\title{
Immune signatures of prodromal multiple sclerosis in monozygotic twins
}

\author{
Lisa Ann Gerdes ${ }^{a, 1}$, Claudia Janoschka ${ }^{b, 1}$, Maria Eveslage ${ }^{c}$, Bianca Mannig ${ }^{b}$, Timo Wirth ${ }^{b}$, \\ Andreas Schulte-Mecklenbeck ${ }^{b}(1)$, Sarah Lauks ${ }^{b}$, Laura Glau ${ }^{d}$, Catharina C. Gross ${ }^{b}$, Eva Tolosa ${ }^{d}$, Andrea Flierl-Hecht ${ }^{a}$,

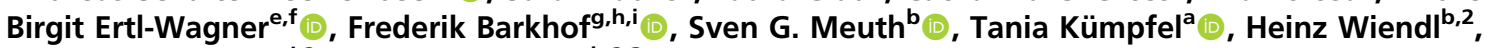 \\ Reinhard Hohlfeld ${ }^{\mathrm{a}, \mathrm{j}, 2}$, and Luisa Klotz ${ }^{\mathrm{b}, 2,3}$
}

\begin{abstract}
anstitute of Clinical Neuroimmunology, University Hospital and Biomedical Center, Ludwig-Maximilians Universität München, 81377 Munich, Germany; ${ }^{b}$ Department of Neurology with Institute of Translational Neurology, University Hospital Münster, Albert-Schweitzer Campus 1, 48149 Münster, Germany; 'Institute of Biostatistics and Clinical Research, University of Münster, 48149 Münster, Germany; ${ }^{d}$ Department of Immunology, University Medical Center Hamburg-Eppendorf, 20246 Hamburg, Germany; ${ }^{e}$ Department of Radiology, Grosshadern Medical Campus, Ludwig-Maximilians Universität München, 81377 Munich, Germany; ${ }^{f}$ Department of Medical Imaging, Hospital for Sick Children, University of Toronto, Toronto, ON M5G 1X8, Canada; ${ }^{9}$ University College London Institute of Neurology, University College London, WC1E 6BT London, United Kingdom; ' University College London Institute of Healthcare Engineering, University College London, WC1E 6BT London, United Kingdom; 'Department of Radiology and Nuclear Medicine, VU University Medical Center, 1105 AZ Amsterdam, The Netherlands; and ${ }^{j}$ Munich Cluster of Systems Neurology, 81377 Munich, Germany
\end{abstract}

Edited by Lawrence Steinman, Stanford University School of Medicine, Stanford, CA, and approved July 28, 2020 (received for review February 21, 2020)

The tremendous heterogeneity of the human population presents a major obstacle in understanding how autoimmune diseases like multiple sclerosis (MS) contribute to variations in human peripheral immune signatures. To minimize heterogeneity, we made use of a unique cohort of $\mathbf{4 3}$ monozygotic twin pairs clinically discordant for MS and searched for disease-related peripheral immune signatures in a systems biology approach covering a broad range of adaptive and innate immune populations on the protein level. Despite disease discordance, the immune signatures of MS-affected and unaffected cotwins were remarkably similar. Twinship alone contributed $56 \%$ of the immune variation, whereas MS explained 1 to $2 \%$ of the immune variance. Notably, distinct traits in $\mathrm{CD}^{+}$effector $\mathrm{T}$ cell subsets emerged when we focused on a subgroup of twins with signs of subclinical, prodromal MS in the clinically healthy cotwin. Some of these early-disease immune traits were confirmed in a second independent cohort of untreated early relapsing-remitting MS patients. Early involvement of effector $T$ cell subsets thus points to a key role of $\mathrm{T}$ cells in MS disease initiation.

multiple sclerosis | monozygotic twins | immune phenotyping | autoimmunity | biomarker

$\mathbf{R}$ esearch into human autoimmunity is facing a number of particular challenges. First, in contrast to inbred animal strains housed under controlled conditions, the human population is exceedingly heterogeneous, with a high degree of genetic and environmental diversity. Second, human autoimmune diseases are usually diagnosed long after initiation of the disease process, so it is impossible to differentiate between early (primary) and late (secondary) immune processes. Third, human studies mostly rely on the blood as the main source of immune cells because access to human tissues is usually limited. Last but not least, ethical constraints limit the scope of functional ("mechanistic") human studies compared to sophisticated animal systems. For these and other reasons it has been very difficult to capture the early footprints ("signatures") of nascent human autoimmune reactions in the blood, the most accessible peripheral immune compartment. This holds particularly true for organspecific, "compartmentalized" autoimmune processes, such as autoimmune diabetes, rheumatoid arthritis, or multiple sclerosis (MS).

MS is a prominent example of such a compartmentalized autoimmune disease as it affects the central nervous system (CNS), which is partly secluded from the peripheral immune system by the blood-brain barrier (1-3). Classic experiments in animals showed that experimental autoimmune encephalomyelitis (EAE), the most widely used model of MS, can be induced in naïve recipient animals by injecting purified myelin autoantigen-specific CD $4^{+}$ effector $\mathrm{T}$ cells $(4,5)$. However, consistent with the excessive heterogeneity of the human population, it has so far not been possible to identify any unifying "target autoantigen" in human MS. There are additional notable differences between EAE models and human MS, including the well-known preponderance of clonally expanded $\mathrm{CD}^{+}$, rather than $\mathrm{CD}^{+}$, T cells in MS brain lesions, and the presence of oligoclonal immunoglobulins in the cerebrospinal fluid (CSF) of MS patients (6-9). Yet despite knowledge about distinct MS-associated features in affected tissues, identification of an MS-associated peripheral immune signature has remained elusive.

Apart from the heterogeneity of the human population, builtin delays in the diagnostic process of autoimmune diseases have hindered the identification of peripheral disease-specific signatures. In MS for example, diagnosis strictly requires the presence of clinical symptoms according to prevailing consensus criteria (10). However, the appearance of symptoms can be preceded by

\section{Significance}

Multiple sclerosis is an autoimmune disease shaped by genetic and environmental factors. Because of the heterogeneity of the human population, it has been difficult to identify "immune signatures" of the disease. Here we investigated a cohort of identical twin pairs who are discordant for multiple sclerosis. In each twin pair, the immune signatures were remarkably similar, pointing to a strong influence of shared genetic and environmental factors. However, when we focused on a subgroup of seemingly healthy cotwins who showed subtle signs of "subclinical neuro-inflammation," we identified a distinct signature of memory T cells. Insight into the immunological mechanisms associated with the initiation of the disease is relevant not only to the therapy but also for prevention of the disease.

Author contributions: L.A.G., C.J., R.H., and L.K. designed research; L.A.G., C.J., B.M., and L.K. performed research; C.J., M.E., B.M., T.W., A.S.-M., S.L., L.G., C.C.G., E.T., A.F.-H., B.E.-W. F.B., S.G.M., T.K., H.W., and L.K. analyzed data; L.A.G. was principal investigator of the MS Twin Study, including organization, recruitment, and patient care; A.F.-H., B.E.-W., T.K., and R.H. were coinvestigators of the MS Twin Study; S.G.M., H.W., and L.K. characterized MS and control cohorts and contributed patient samples; and L.A.G., C.J., and L.K. wrote the paper.

The authors declare no competing interest.

This article is a PNAS Direct Submission.

This open access article is distributed under Creative Commons Attribution-NonCommercialNoDerivatives License 4.0 (CC BY-NC-ND).

See online for related content such as Commentaries.

'L.A.G. and C.J. contributed equally to this work.

${ }^{2}$ H.W., R.H., and L.K. contributed equally to this work.

${ }^{3}$ To whom correspondence may be addressed. Email: luisa.klotz@ukmuenster.de.

This article contains supporting information online at https://www.pnas.org/lookup/suppl/ doi:10.1073/pnas.2003339117/-/DCSupplemental.

First published August 17, 2020. 
a long-lasting prodromal phase of clinically silent neuroinflammation. This is detected only very rarely and usually only by chance when unexpected silent brain lesions are observed on MRI performed for an unrelated medical reason, a setting referred to as "radiologically isolated syndrome" (11).

In searching for peripheral MS-associated immune signatures, we here took advantage of a unique cohort of 43 monozygotic twin pairs who are discordant for MS: That is, one cotwin in each pair carries the diagnosis of MS, whereas the other cotwin is clinically healthy. We reasoned that pairwise comparisons of these monozygotic twins would eliminate genetic heterogeneity for each pair, and in addition reduce environmental heterogeneity as the twins included in this study were raised in the same household. In a first step of analysis, we found that the immune signatures of MS-affected and unaffected cotwins were remarkably similar, whereas age, sex, and MS disease status together contributed less than $10 \%$ of the variation of immune traits. In a second step, we leveraged another distinct advantage of the MSdiscordant twin cohort: The healthy cotwins of MS-affected twins have a maximum familial risk of developing MS and therefore an increased probability of showing signs of prodromal MS (12). Indeed, several clinically unaffected cotwins showed distinct signs of early subclinical neuroinflammation (SCNI) on MRI. By focusing on these subjects with early neuroinflammation, we were able to detect an early influence of MS on the peripheral immune signature, using high-resolution flow cytometry-based immune phenotyping followed by unbiased machine-based analysis and dimensionality reduction algorithms.

\section{Results}

High Degree of Similarity in Peripheral Immune Signatures within Twin Pairs Despite Disease Discordance. In this study, we evaluated MSrelated changes in peripheral immune signatures in a cohort of 43 monozygotic twin pairs clinically discordant for MS (Fig. 1A), as a twin approach offers the important advantage to control for genetic as well as intrinsic factors as age and sex, as well as early environmental factors. All 43 monozygotic twin pairs were of Caucasian origin and were raised together until adulthood and shared a variety of early environmental factors. Demographic data, as well as information with regard to MS disease course and treatment, are depicted in Table 1 . We took advantage of a comprehensive deep functional immune-cell phenotyping platform by using multicolor flow cytometry on frozen peripheral blood mononuclear cell (PBMC) specimens. In this system immunology approach, 13 9- to 10-color immunophenotyping panels were used for the characterization of major immune cell subpopulations and their
A
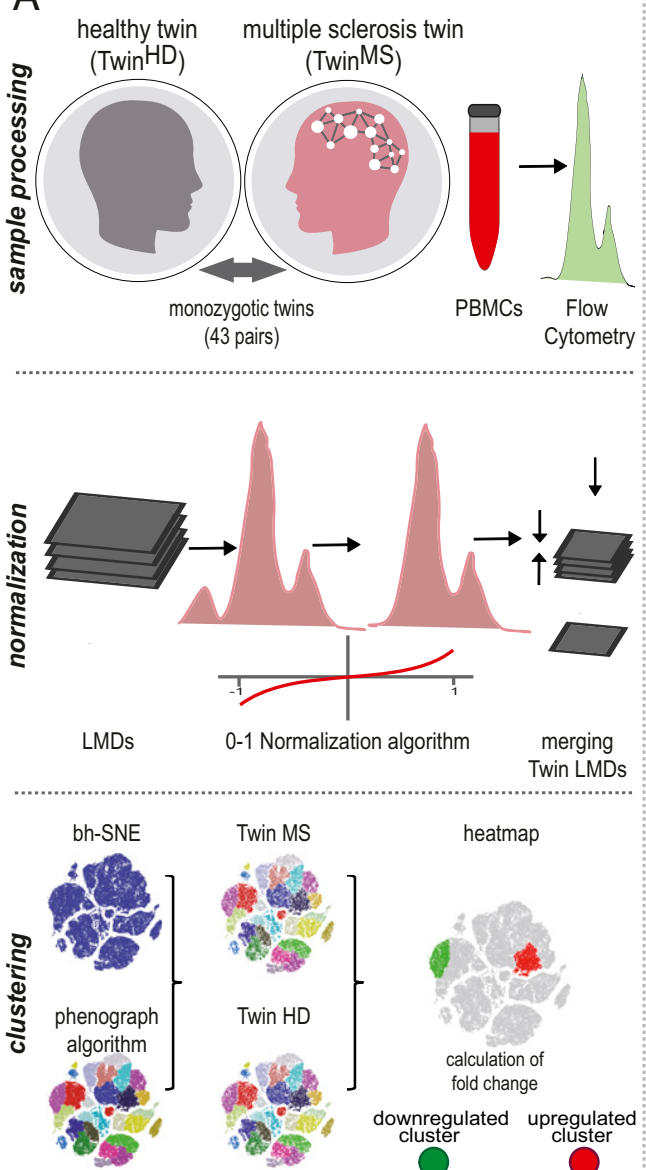

B
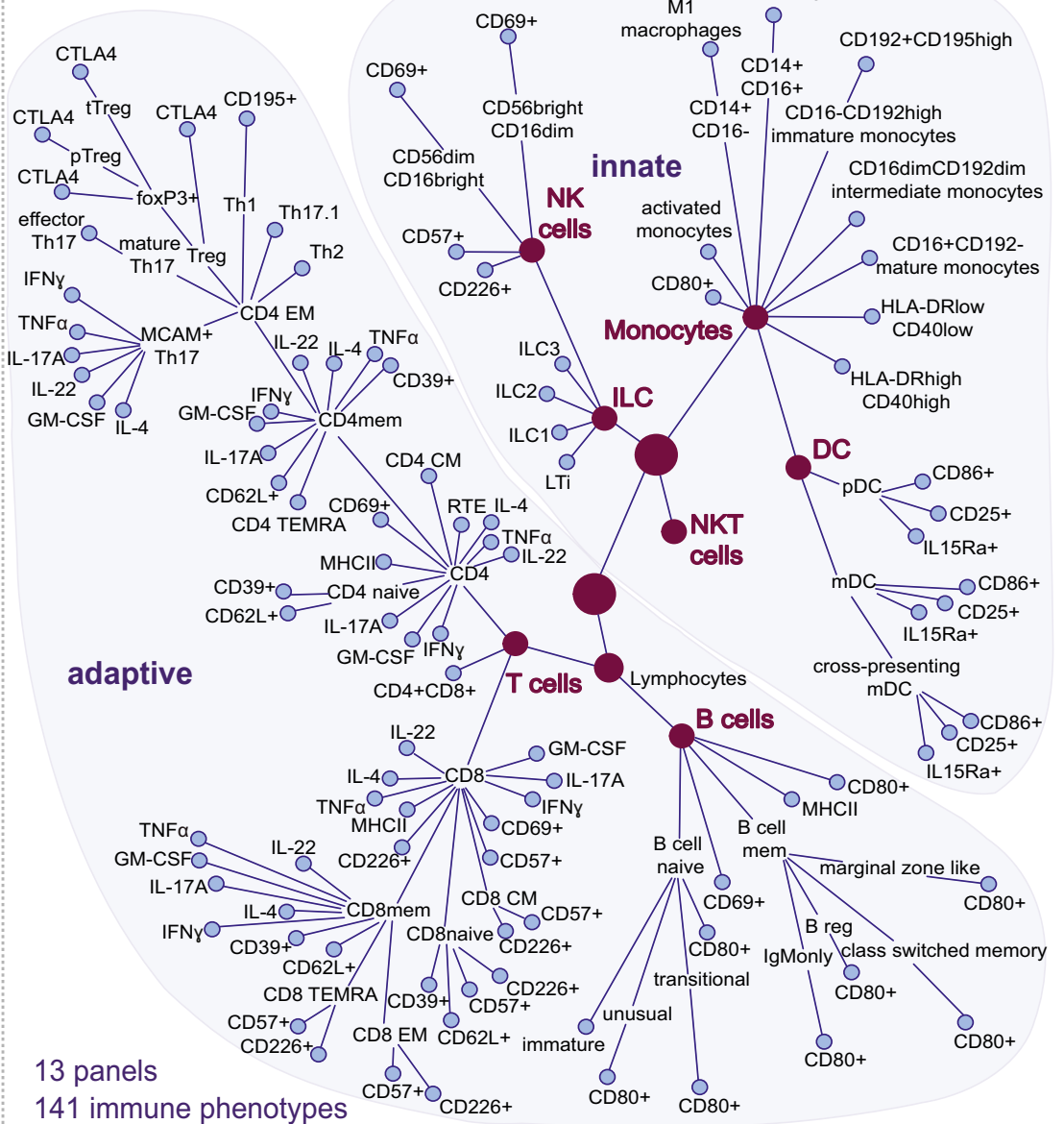

13 panels
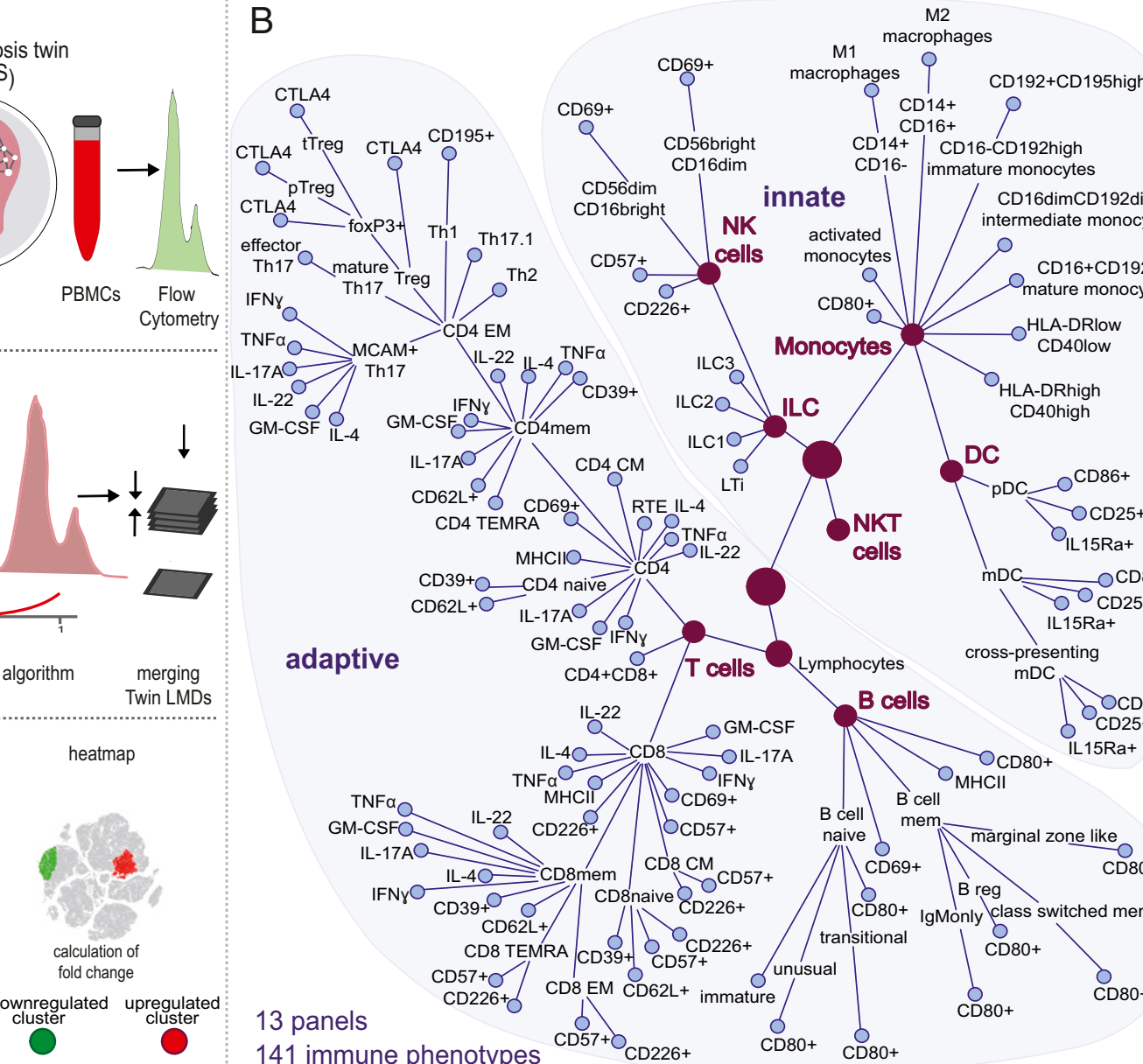

innate

CD14+ CD16-CD192high

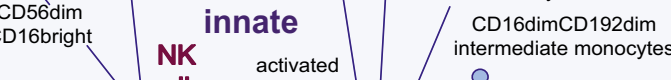

CD57+

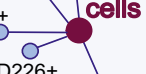

monocytes 0

CD80+ Omature monocytes

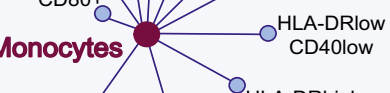

(
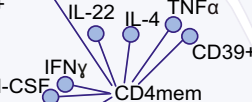

ILC2O ILC

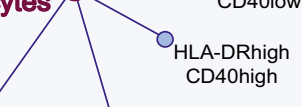

Fig. 1. In-depth characterization of monozygotic twins discordant for MS. (A) Twin study set-up of monozygotic twin pairs ( $n=43$ ) discordant for MS: Healthy (TWIN ${ }^{\mathrm{HD}}$, gray) and MS (TWIN ${ }^{\mathrm{MS}}$, red) twin. Sample processing; cryoconserved PBMC of study participants were processed via flow cytometry in 13 panels of 10 colors each. For unbiased nonlinear dimensionality reduction via bh-SNE, LMDs were transformed, normalized, and merged per panel and randomly subsampled in viSNE, an implementation in Matlab. For visual representation of single-cell data and identification of population clusters, a PhenoGraph algorithm was used; fold-change was calculated per cluster comparing compiled data from all Twin ${ }^{\mathrm{HD}}$ to Twin $^{\mathrm{MS}}$; a regulation greater than twofold (up-regulation in red, down-regulation in green) is visualized in a heatmap. (B) Hierarchical illustration of the defined populations ( $n=141)$ analyzed within this study. Red dots illustrate the respective main populations within the adaptive and innate compartment, further subpopulations are indicated by blue dots. 
maturation and functional status $(13,14)$. Longitudinal repeated measurements $(n=26)$ of PBMCs derived from a study-independent healthy individual during the total acquisition period of $4 \mathrm{wk}$ (mean variability of traits $r=0.05$ ) revealed robustness of data acquisition (Dataset S4). To accommodate for sample-to-sample acquisition variability, we used a linear normalization algorithm and applied it on a single-file level. The normalized single-cell data were then visualized by Barnes-Hut stochastic neighbor embedding (bh-SNE) and PhenoGraph, a machine-based algorithm for clustering and dimensionality reduction of high-dimensional single-cell data $(15,16)$. Fig. $1 B$ illustrates the immune cell parameters as determined by conventional gating, finally encompassing 141 different immune phenotypes, which we here refer to as immune cell traits (17).

To detect and visualize distinct immune signatures in healthy twins (TWIN ${ }^{\mathrm{HD}}$ ) (Fig. $2 A$, first row) and MS-affected (TWIN ${ }^{\mathrm{MS}}$ ) (Fig. $2 A$, second row) cotwins, we used unsupervised clustering to identify major immune cell populations within each panel (Fig. $2 A$ ) by the PhenoGraph algorithm. The algorithm identified 30 distinct immune cell clusters (as described in Materials and Methods) in high-dimensional space based on compiled TWIN $^{\mathrm{HD}}$ and TWIN ${ }^{\mathrm{MS}}$ single-cell data. We did not observe any differences between TWIN ${ }^{\mathrm{HD}}$ and TWIN ${ }^{\mathrm{MS}}$ in any of the immune cell traits encompassed by this analysis as shown in the heatmaps (Fig. $2 A$, third row). This was corroborated by conventional manual gating approaches yielding the frequencies of the main immune cell populations and subsequent Pearson correlation analysis (Fig. $2 A$ and $S I$ Appendix, Fig. S1 $A$ ). Notably, also pairwise comparison within individual twin pairs revealed a remarkable similarity in the immune traits for all major immune cell populations, with a slight tendency for differential patterns only found in the B cell subset of treated MS cotwins (Fig. 2B).

We next analyzed immune cell lineages in more detail, by considering all $(n=13)$ (Fig. $2 C)$ immunophenotyping panels together. Across all flow cytometry panels, PhenoGraph identified 352 discrete immune cell clusters in high-dimensional space, which we used to reveal potential differences between TWIN $^{\mathrm{HD}}$ and TWIN $^{\mathrm{MS}}$ immune cell signatures with higher resolution. Still, we did not observe any distinctly emerging changes (i.e., defined as more than twofold) per cluster between TWIN ${ }^{\mathrm{HD}}$ and TWIN $^{\mathrm{MS}}$, again pointing toward a strong similarity within each twin pair (Fig. $2 C$ and $D$ ).
When focusing our analysis on those twin pairs without any MS treatment-to exclude potential treatment-related confounding factors-we again did not detect any relevant changes within each cluster comparing TWIN ${ }^{\mathrm{HD}}$ to $\mathrm{TWIN}^{\mathrm{MS}}$ groups (SI $A p$ pendix, Fig. $\mathrm{S} 1 B$ ).

We also performed functional analyses of major subsets, using intracellular cytokine expression analysis following short-term stimulation. We found slightly lower frequencies of cytokineproducing $\mathrm{CD}^{+}$and $\mathrm{CD}^{+} \mathrm{T}$ memory subsets in the MS cotwins; however, this difference was completely abolished when focusing on those MS cotwins not receiving any immune therapy at the time of blood sampling, hence suggesting that these effects were most likely treatment related (SI Appendix, Fig. S1C).

Twinship Explains the Majority of the Variance in Peripheral Immune Traits. We next aimed to decipher and quantify the distinct individual effects of defined factors, such as twinship, MS status, age, gender, and cytomegalovirus (CMV) serostatus on the variation of all innate and adaptive immune cell traits investigated using a linear mixed-model approach (Fig. 3A). The factor "twinship" encompasses both the genetic background, and shared local environmental factors, such as childhood infections or diet, that play a major role in early development of immunity $(18,19)$. Importantly, twinship explained the vast majority of the variance for each of the 141 investigated immune-cell traits, its influence ranging from 11 to $89 \%$ of the explained variance (Fig. $3 B$ ). In contrast, age, sex, CMV, and MS status taken together had a minor contribution $(11 \%)$ to the variance of each parameter (Fig. $3 B$ and $C$ and SI Appendix, Fig. S2). Overall, twinship was responsible on average for the major proportion $(56 \%)$ of the variance, whereas the MS status explained $1 \%$, age $4 \%$, CMV status $3 \%$, and sex $2 \%$ of the proportion of the variance of immune traits (Fig. 3D). To search for subtle differences in the contribution of each factor, all parameters were grouped according to their immunological "ancestry" (Fig. 1B). Again, the MS status alone explained minor proportions of the variation, ranging from $1 \%$ in conventional $\mathrm{T}$ cells and innate immune cells, to $2 \%$ in B cells, monocytes and dendritic cells (DC) (Fig. $3 C$ and $D)$. Interestingly, the contribution of twinship to the variance of B cell traits was slightly less pronounced (Fig. $3 C$ and $D$ ), albeit

Table 1. Basic clinical characteristics of patient cohorts

\begin{tabular}{|c|c|c|c|c|c|}
\hline & $\begin{array}{l}\text { Monozygotic } \\
\text { twin pairs }\end{array}$ & $\begin{array}{l}\text { Monozygotic twin } \\
\text { pairs with SCNI } \\
\text { in healthy cotwin }\end{array}$ & $\begin{array}{l}\text { Clinically solated } \\
\text { syndrome }\end{array}$ & $\begin{array}{l}\text { Treatment naïve } \\
\text { early MS (RRMS) }\end{array}$ & $\begin{array}{l}\text { Control cohort } \\
\text { (HD) }\end{array}$ \\
\hline Number & 43 & 10 & 55 & 60 & 71 \\
\hline $\operatorname{Sex}(f / m)$ & $33 / 10$ & $8 / 2$ & $43 / 12$ & $43 / 17$ & $44 / 27$ \\
\hline Age (mean, range) & $43.7(20-67)$ & $44(20-67)$ & $32.6(18-56)$ & $33.1(18-55)$ & $33.3(21-56)$ \\
\hline \multicolumn{6}{|l|}{ Disease course } \\
\hline RRMS & 26 & 7 & 0 & 60 & NA \\
\hline CIS & 3 & 1 & 55 & 0 & NA \\
\hline SPMS & 12 & 2 & 0 & 0 & NA \\
\hline PPMS & 2 & 0 & 0 & 0 & NA \\
\hline Disease duration & 14 y $(0.5-45)$ & 14 y $(1-30)$ & $1.6 \mathrm{mo}(0-10)$ & $2.7 \mathrm{mo}(0-10)$ & NA \\
\hline EDSS & $1.7(0-9.5)$ & $3.2(1-8.5)$ & $1.3(0-2.5)$ & $1.3(0-4.5)$ & NA \\
\hline Disease modifying treatment & & & & & NA \\
\hline None & 22 & 5 & 55 & 60 & NA \\
\hline IFN- $\beta$ & 14 & 4 & 0 & 0 & NA \\
\hline Glatiramer acetat & 3 & 0 & 0 & 0 & NA \\
\hline Natalizumab & 4 & 1 & 0 & 0 & NA \\
\hline EBV status $(+/-)$ & $71 / 1 *$ & $20 / 0$ & & & NA \\
\hline CMV status $(+/-)$ & $31 / 53^{\dagger}$ & $10 / 10$ & & & NA \\
\hline
\end{tabular}

NA, not applicable; PPMS, primary progressive MS; SPMS, secondary progressive MS

*Tested pairs $n=36$.

${ }^{\dagger}$ Tested pairs $n=42$. 

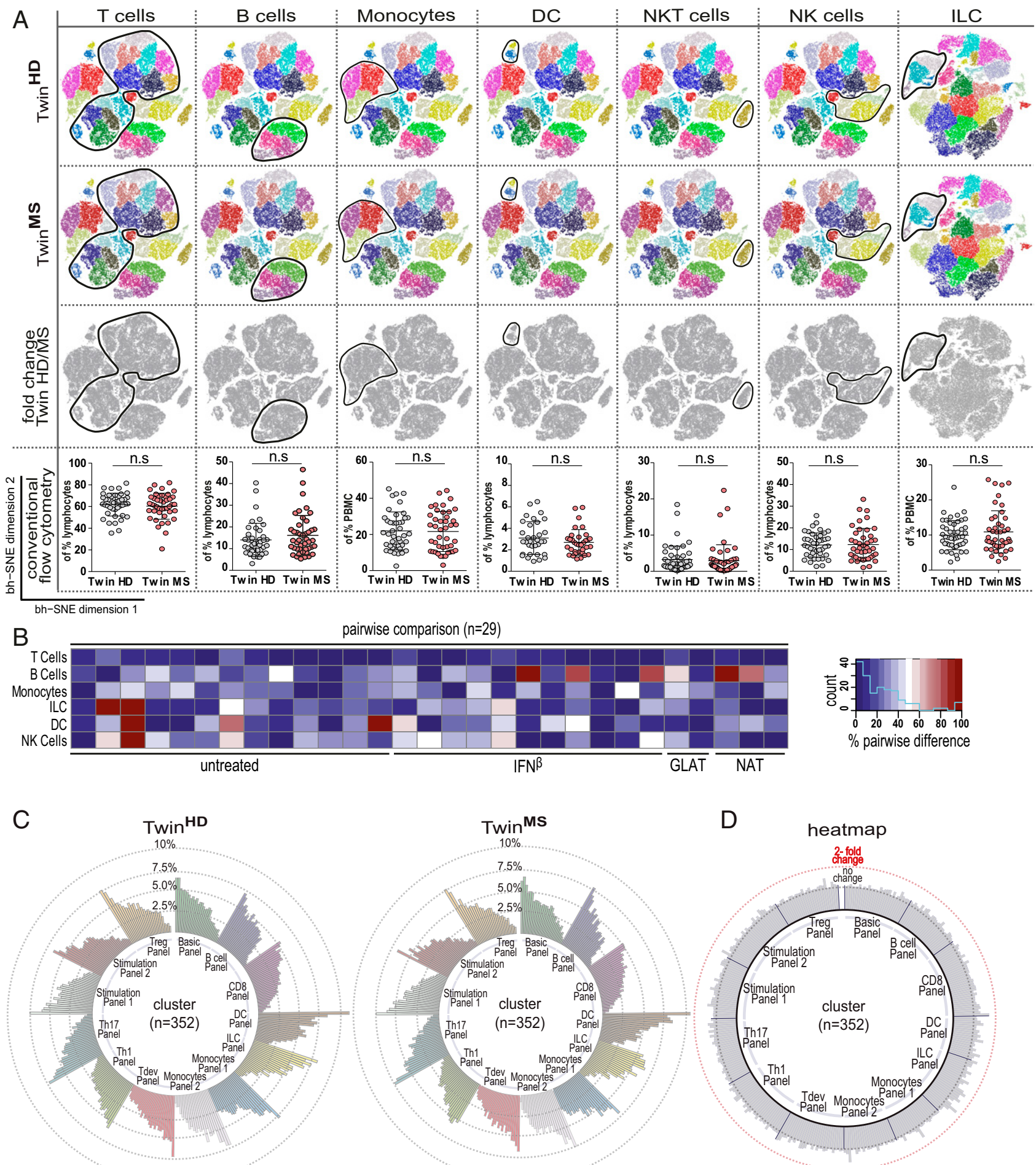

Fig. 2. Frequencies of immune cell populations and functional properties in monozygotic twins discordant for MS. (A) PhenoGraph-maps compare single cell clusters of major immune cell populations of compiled TWIN ${ }^{\mathrm{HD}}$ (row 1$)$ to TWIN ${ }^{\mathrm{MS}}$ (row 2 ) data indicated by black circles (basic panel $n=43$ pairs; ILC panel $n=$ 34 pairs). Heatmap (row 3 ) illustrates the fold-change per cluster between TWIN ${ }^{\text {MS }}$ and TWIN ${ }^{\text {HD; }}>>$ twofold up-regulation (red) and $>$ twofold down-regulation (green) would be indicated within the black circles. Corresponding conventional flow cytometry data comparing TWIN ${ }^{\mathrm{HD}}$ to $\mathrm{TWVIN}^{\mathrm{MS}}$ are depicted in row 4. (B) Illustration of pairwise differences within individual twin pairs for all major immune populations based on conventional flow cytometry data; heatmap illustrates the difference in percentage. Immune therapy of MS twins (if applicable) is indicated below, IFN- $\beta$, natalizumab (NAT), and glatiramer acetate (GLAT), or no treatment (untreated). (C) Unbiased single-cell analysis of flow cytometry data via viSNE for each individual panel (panels = 13); PhenoGraph algorithm identified in total 352 clusters of compiled data from TWIN ${ }^{\mathrm{HD}}$ and TWIN ${ }^{\text {MS }}$ groups (basic-, Th1-, Th17-, stimulation 2 panel $n=43 ;$ B cell panel $n=41$ pairs; CD8 $8^{+}$panel $n=39$ pairs; DC panel $n=30$ pairs; ILC panel $n=34$ pairs; monocyte panel $1+2 n=25$ pairs; Tdev panel $n=37$ pairs; stimulation 1 panel $n=$ 31 pairs; Treg panel $n=42$ pairs); illustrations display the frequency per cluster within each panel. $(D)$ Corresponding heatmap illustrates the fold-change between TWIN ${ }^{\mathrm{MS}}$ and TWIN ${ }^{\mathrm{HD}}$ groups for each individual cluster; twofold change line (dotted red line), no change line (dotted gray line) are indicated, Graph values represent mean \pm SD. Statistical significance was evaluated by Wilcoxon matched-pairs signed rank test; n.s.: not significant. 


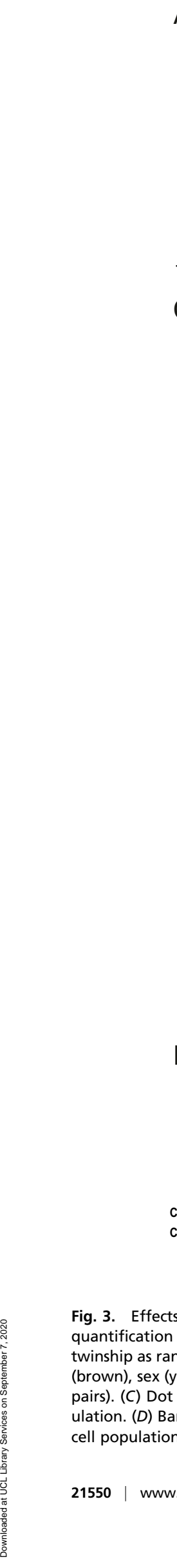

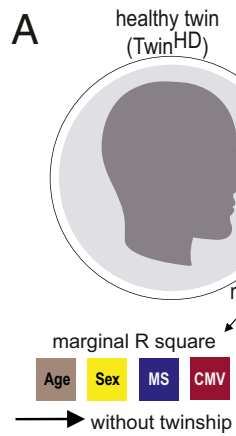
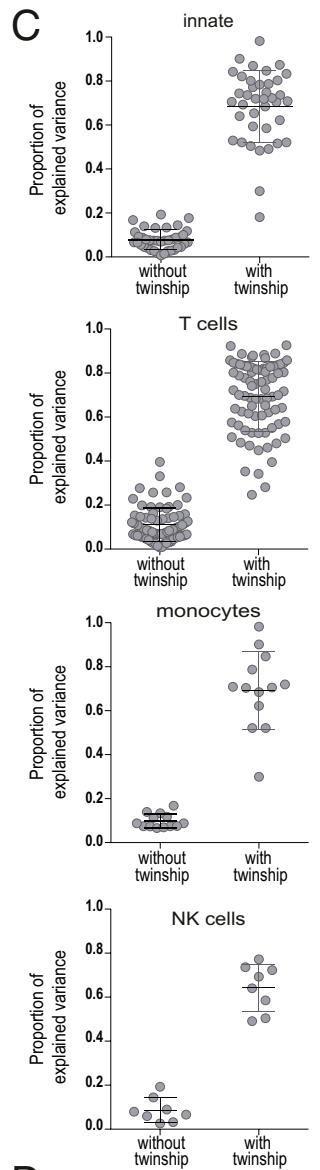

D

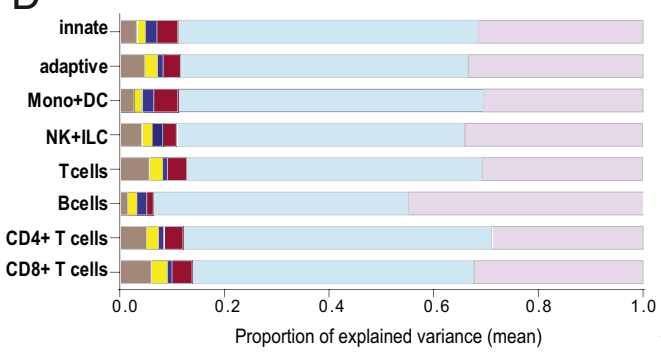
multiple sclerosis twin sclerosis
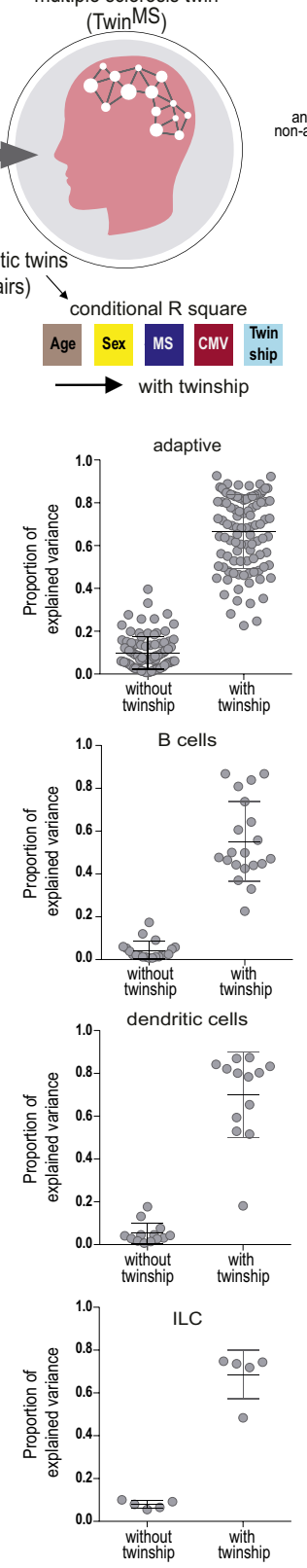

B

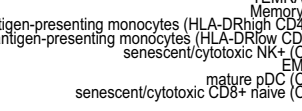

$$
\begin{aligned}
& \begin{array}{r}
\text { homing L-Selectin } \mathrm{CD} 62 \mathrm{~L}+(\mathrm{CD} 4+\mathrm{CD} D \\
\text { ectoenzyme } \mathrm{CD} 39+\mathrm{CD} 8+\mathrm{CD}+
\end{array} \\
& \begin{array}{r}
\mathrm{CD} 8 \mathrm{mem+} \\
\mathrm{CD} 5 \mathrm{dim} \mathrm{CD} 16+\mathrm{N} \\
\mathrm{CD} 4+\mathrm{G}
\end{array}
\end{aligned}
$$

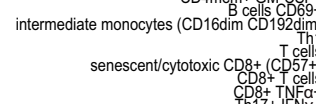

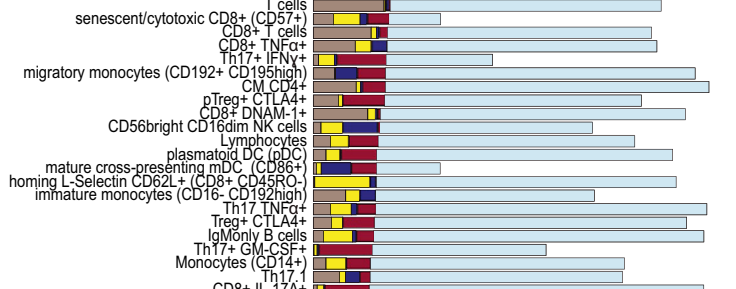

homing L-Selectin CD62L+ $(\mathrm{CD} 8+\mathrm{CD} 8+1 \mathrm{C}-17 \mathrm{C}$.

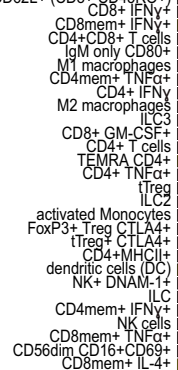

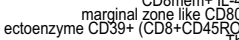
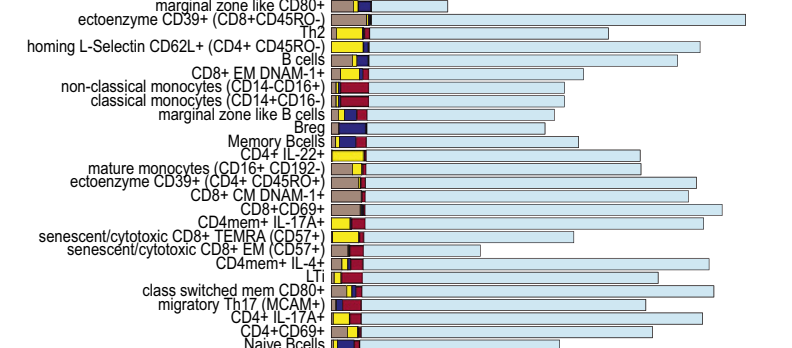

ND4+CD69+
Naive BCells
effector Th17 (CCR4+CCR6+CD161+MCAM+)

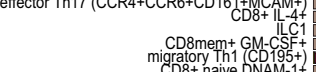

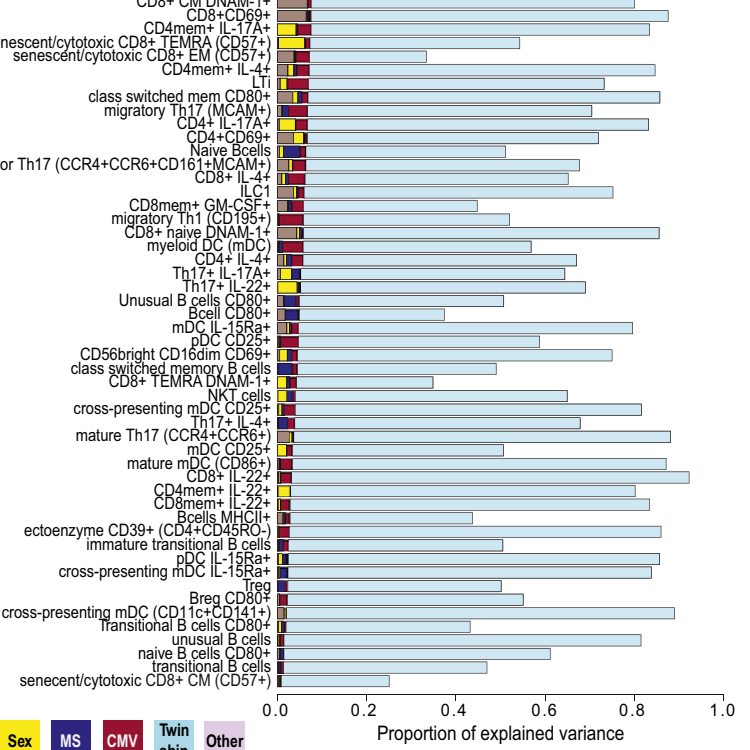

Sex MS CMv $\begin{gathered}\text { Twin } \\ \text { ship }\end{gathered}$ Othe

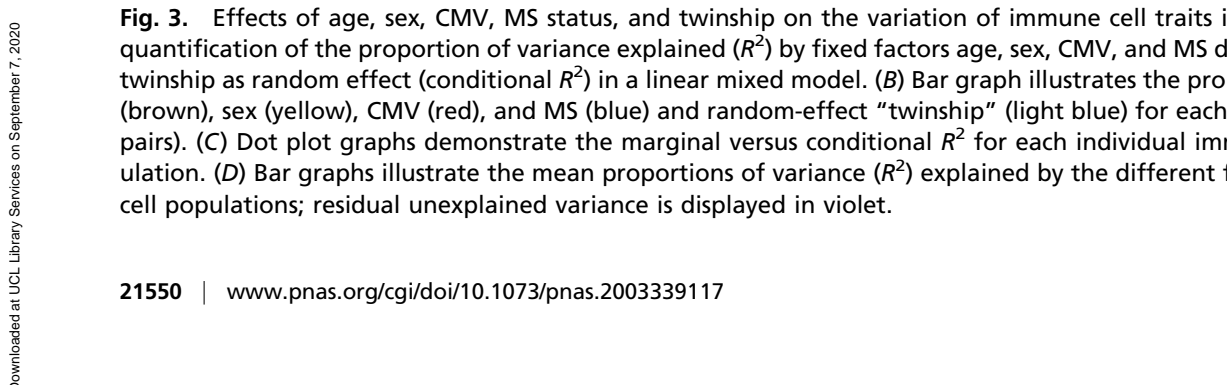


still high, with $55 \%$ of the explained variance, suggesting that B cell traits might be less stringently genetically controlled as compared to other immune traits.

Identification of MS-Related Immune Traits by Analysis of Cotwins with Subclinical Neuroinflammation. It has been shown that individuals with an increased risk of MS based on a combined genetic and environmental risk are more likely to have early subclinical manifestations of MS (20). Specifically, in our twin cohort the clinically healthy cotwins have a maximum familial risk of developing MS. We therefore applied detailed investigations, including cerebral imaging (cMRI) and CSF analysis, to detect potential SCNI in the clinically unaffected cotwins. We defined SCNI as the presence of either cMRI lesions suggestive of MS, rated by a blinded MS neuroradiologist, or detection of CSF-specific oligoclonal bands indicative of intrathecal $\mathrm{IgG}$ synthesis (8). A significant proportion of clinically unaffected cotwins $(n=10)$ presented signs of SCNI, suggesting the presence of prodromal MS. A group of clinically healthy cotwins $(n=$ 14) without any signs of SCNI were used as controls for further analysis (Fig. 4A). We first repeated our unbiased cluster analysis of healthy twins versus MS twins by only incorporating datasets from healthy twins without any signs of SCNI; however, this did not result in the identification of differentially regulated clusters (SI Appendix, Fig. S3). We then hypothesized that if any immune cell trait is indeed influenced by MS, the extent of its correlation would be higher within SCNI-MS twin pairs than in healthy donor (HD)-MS twin pairs based on the premise that SCNI represents the earliest detectable stage of MS. We therefore performed a Spearman correlation analysis adjusted for MS status, comparing the correlation coefficient of SCNI-MS twin pairs with the correlation coefficient of HD-MS twin pairs for all immune cell subsets $(n=141)$ (SI Appendix, Fig. S4A). As hypothesized, we observed a significant increase in the degree of correlation in SCNI-MS twin pairs particularly within the adaptive compartment (i.e., all T cells and, more specifically, in $\mathrm{CD} 4^{+}$and $\mathrm{CD}^{+} \mathrm{T}$ cell subsets). The degree of correlation was also slightly increased for the monocyte/dendritic cell subset.

To further corroborate these findings by another statistical approach, we calculated the intraclass correlation coefficient (ICC) (Fig. 4B) for each immune cell trait based on a linear mixed-model adjusted for MS, and corrected the comparison between SCNI-MS and HD-MS twin cohorts for the hierarchical dependency of parameters (SI Appendix, Fig. S4B). The advantage of this approach is that the mixed model was additionally adjusted for the interdependence of immune parameters as described in Materials and Methods. Comparison of the ICC of the major immune cell populations revealed a significant increase in the SCNIMS twin pairs compared to the HD-MS twin pairs for T cells but neither for B cells nor the major innate cell populations (Fig. $4 B$ and SI Appendix, Fig. S4C), again supporting the concept that the degree of correlation is enhanced in SCNI-MS twin pairs as compared to HD-MS twin pairs. Within the T cell compartment, we observed a significant ICC rise in the $\mathrm{CD} 4^{+}$but not $\mathrm{CD} 8^{+}$ T cell subsets (Fig. $4 B$ and SI Appendix, Fig. S5A).

For further corroboration of this concept we made use of unbiased t-distributed stochastic neighbor embedding ( $t-S N E$ ) dimensionality reduction of our flow cytometry data to calculate the biological distance within each individual twin pair as an indicator of the degree of similarity within predefined $\mathrm{CD}^{+}{ }^{+} \mathrm{T}$ cell subsets as described in Materials and Methods (a representative example of such a calculation is depicted in Fig. $4 C$ and SI Appendix, Fig. S5B). Indeed, we observed a significant difference in the biological distance between SCNI-MS twin pairs and HD-MS twin pairs for $\mathrm{CD}^{+}$effector subsets (Fig. 4C), again pointing toward a higher degree of similarity within the SCNI-MS twin pairs. Heatmap visualization of pairwise differences (in percent) for individual parameters within the $\mathrm{CD} 4^{+}$effector subsets further illustrated the increased degree of similarity within the SCNI-MS twin pairs, which was particularly pronounced within proinflammatory $\mathrm{CD}^{+}{ }^{+}$T-cell subsets, such as Th17 and Th1 cell populations (Fig. 4D).

Validation of MS-Related Immune Traits in an Early MS Replication Cohort. Finally, we wanted to determine whether our approach of analyzing individuals with prodromal MS might facilitate identification of potentially disease-associated peripheral immune signatures in a replication cohort of subjects with either clinically isolated syndrome (CIS) or early clinically manifest relapsingremitting MS (RRMS) according to the 2010 Mc Donald criteria (21), that were all treatment-naive at the time of blood sampling (Fig. $4 E$ ). In this cohort, biosample acquisition took place within the first $3 \mathrm{y}$ from the time of clinically defined disease onset. We were able to obtain flow cytometry data of our key candidate populations identified in Fig. $4 D$, in 55 patients with clinically isolated syndrome and 60 treatment-naïve early RRMS patients, as well as 71 age- and sex-matched healthy controls (Table 1). Indeed, conventional flow cytometry analysis revealed significant MS-related changes in some of the previously identified immune traits from our twin approach, such as Th1 cells, migratory Th17 cells $\left(\mathrm{MCAM}^{+}\right)$, effector Th17 cells, migratory Th1 cells $\left(\mathrm{CD} 195^{+}\right)$, as well as Th17.1 cells (Fig. $4 E$ and SI Appendix, Fig. S5C). Notably, at least some of these changes were slightly more pronounced in the CIS cohort, such as effector Th17 cells and migratory Th17 cells, again supporting the concept of early disease-related changes in peripheral immune signatures in the course of disease evolution.

\section{Discussion}

In our study we aimed at capturing autoimmunity-related changes in the peripheral immune system in a systems biology approach. To this end, we took advantage of a MS-discordant monozygotic twin cohort, which allows for controlling genetic as well as intrinsic factors, including shared early environmental influences on immune cell traits. At the level of the overall cohort, immune signatures of MS-affected and unaffected twins were remarkably similar despite discordance for an autoimmune disease. However, when we focused on a subgroup of clinically healthy cotwins showing signs of early subclinical neuroinflammation, a distinct MS-related immune signature emerged.

Dominance of Twinship Over MS Disease Status. Using a comprehensive flow cytometry-based immune phenotyping approach and linear mixed models, we determined the relative impact of age, sex, CMV status, MS status, and twinship on the variation of each immune cell trait. Our analysis revealed that the impact of twinship was dominant, ranging from $11 \%$ to a maximum $89 \%$, with a mean of $56 \%$ of explained variance for all immune traits investigated. Other known factors - such as age, sex, and CMV status - had an influence on average of $4 \%$ for age, $2 \%$ for sex, and $3 \%$ for CMV, thus confirming their acknowledged effects on cellular immune compartments as, for example, recent thymic emigrant $\mathrm{CD}^{+} \mathrm{T}$ cells for age $(18,22)$. In comparison, MS exerted an average influence of $1 \%$, indicating a similar degree of impact as compared to the established factors age, sex, and CMV status.

A distinct influence of twinship on the variation of immune cell traits was expected based on studies in healthy individuals, especially in light of the known impact of genetic as well as local environmental factors on the immune system (18, 19, 23-26). Along this line, several recent studies provided evidence of a strong genetic influence on immune cell traits: One study revealed that adaptive immune traits-in particular $\mathrm{CD}^{+}$and $\mathrm{CD} 8^{+}$ $\mathrm{T}$ cells-were more influenced by genetics than innate traits (23). Another study also revealed a substantial impact of genetic factors on immune traits in a cohort of 1,620 Sardinians (27). Moreover, an analysis from the human functional genomics project demonstrated that within the lymphocyte population, genetics explained a higher percentage of variation for $\mathrm{T}$ cells (i.e., 30\%) than for 
A

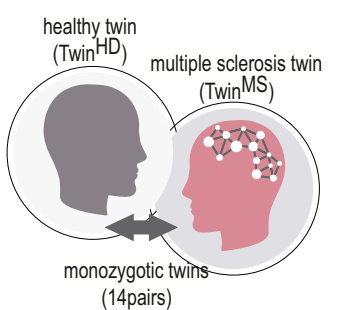

subclinical neuroinflammation twin

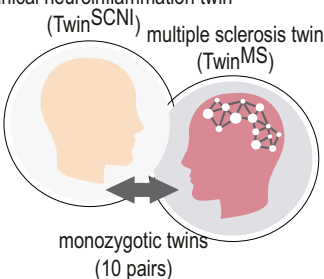

C

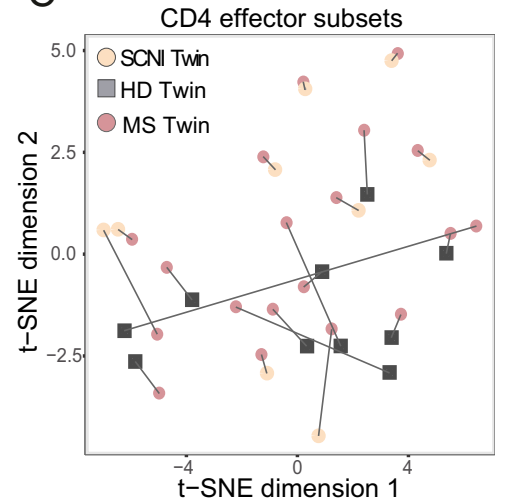

CD4 effector subsets

$\mathrm{t}$-SNE dimension 1
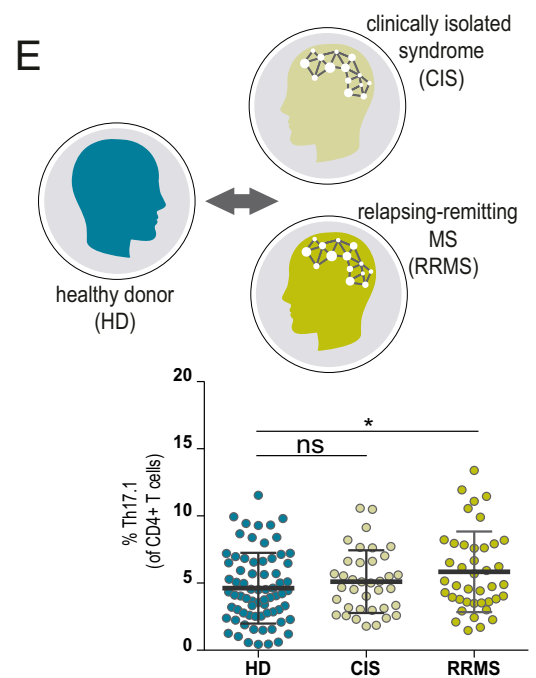
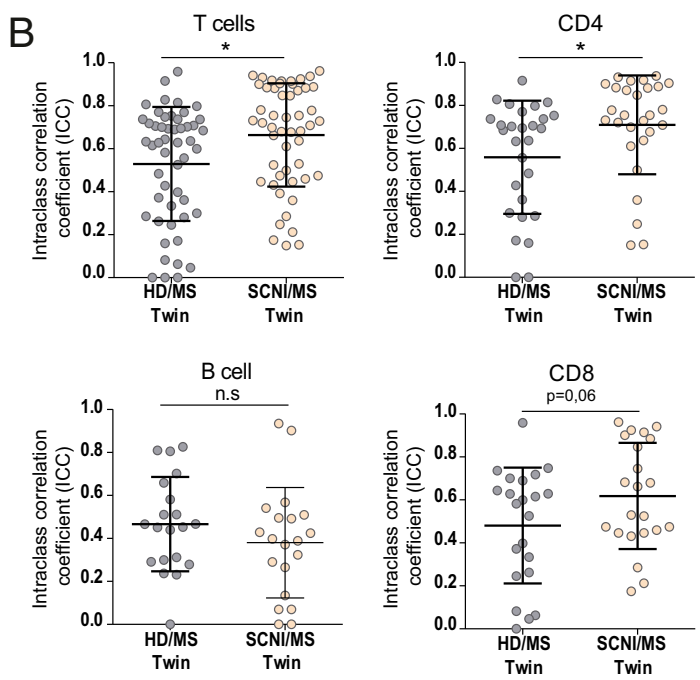

\%pairwise difference

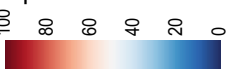

D
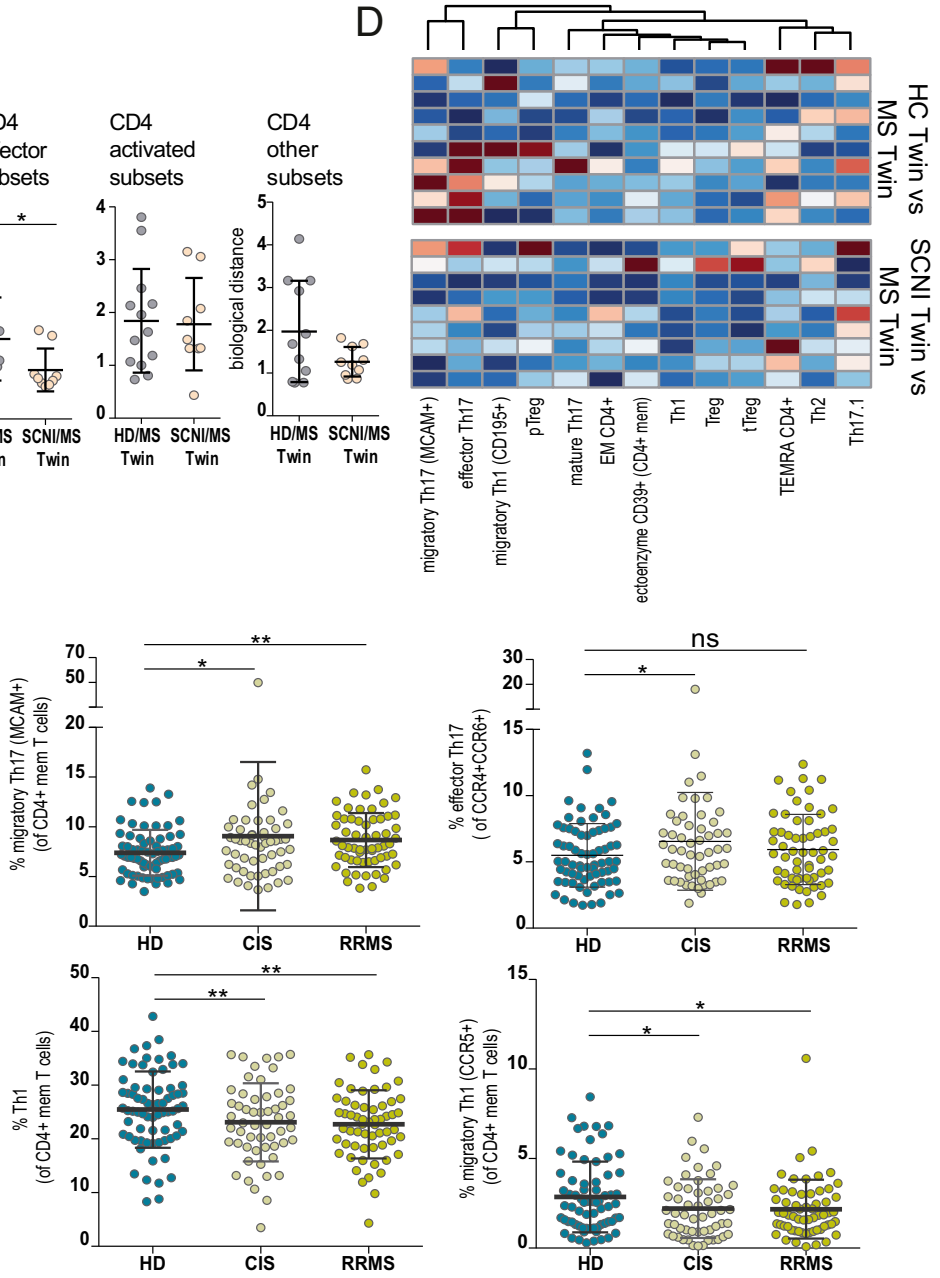

Fig. 4. SCNI analysis identifies effector CD4 populations as differentially regulated populations in the initiation of MS. (A) Illustration of cohort division into SCNI-MS twin pairs ( $n=10$, beige) and HD-MS twin pairs $(n=14$, gray) as described in Materials and Methods. (B) Calculation of the ICC for each immune trait in SCNI-MS twin pairs ( $n=10$, beige) and HD-MS twin pairs ( $n=14$, gray) based on linear mixed model adjusted for MS. To account for hierarchical dependencies between immune cell populations, analyses incorporate corrections using correlation matrixes per sub collective, as described in Materials and Methods. (C) Representative example of t-SNE dimensionality reduction of conventionally analyzed flow cytometry populations comparing the biological distance (gray line) between HD-cotwins (gray rectangle, $n=10$ ) and SCNI cotwins (beige circle, $n=9$ ) to their respective MS cotwin (red circle), for CD4 effector subset ( $n=13$ parameters). $(D)$ Hierarchically clustered heatmap represent the difference in percent for each individual immune cell trait between HD-MS twin compared to SCNI-MS twin within each pair for the CD4 ${ }^{+}$effector subset. (E) Independent validation cohort consisting of healthy controls (HD, $n=71$; turquoise), CIS ( $n=55$, light olive) and treatment naïve MS patients (MS, $n=60$; olive) of selected CD4 ${ }^{+}$effector parameters. Statistical significance was evaluated by linear mixed models, as described in Materials and Methods and Mann-Whitney $U$ test or unpaired $t$ test; ${ }^{*} \leq 0.05 ; * \star P \leq 0.01 ;$ ns: not significant. 
B cells (i.e., 18\%) (24). Although the reasons for this differential influence have not been fully elucidated, the strong association between HLA background and T cell receptor repertoire may contribute to these findings (28). In addition to these observations highlighting the impact of genetics, a large twin study in healthy individuals revealed that also nonheritable influences have a distinct impact on immune traits as illustrated by the profound effect of a latent CMV infection affecting more than $50 \%$ of all immune cell parameters investigated (25). These findings prompted us to include CMV serostatus as fixed factor in our modeling, and indeed, CMV serostatus explained up to $17 \%$ of immune cell parameter variance in our dataset. Along this line, a Belgian survey using a large cohort of healthy individuals provided evidence of a profound impact of local environmental influences as reflected by cohabitation of nonrelated individuals (i.e., parents), which resulted in a $50 \%$ reduction in immunological variation between nonrelated individuals (18). Some of the nonheritable influences might lead to MS-associated epigenetic changes in immune cells, but these have yet to be characterized in detail (29).

In our study, the observed strong effect of twinship is most likely driven by a combined effect of genetic and shared environmental influences because of the fact that all twin pairs in our study were raised together until adulthood. Potentially relevant shared environmental influences range from infections to dietary influences and their respective effects on individual microbiome composition to lifestyle factors, such as exercise, smoking, and alcohol consumption. Importantly, many of these factors not only have been identified as modulators of immune cell traits but have also been implicated in the development of autoimmunity. Particularly in MS, there is evidence that at least some environmental influencing factors not only impact disease incidence and disease course, but also elicit changes in immune signatures $(30,31)$.

An important aspect that might have contributed to the moderate influence of MS at the level of the whole cohort is the relative heterogeneity of the MS-affected cotwins with regard to disease duration (range 0.5 to $45 \mathrm{y}$ ), disease course (relapsing form $67 \%$, progressive form $33 \%$ ), disease severity (expanded disability status scale [EDSS] range 0 to 9.5), as well as immune modulatory treatment (Table 1). In light of the obvious difficulties in the recruitment of monozygotic twins discordant for MS, it was not possible to stringently recruit only early untreated MS twins, although this might have reduced confounding effects due to MS heterogeneity.

MS-Related Signatures in Cotwins with Prodromal MS. To overcome this limitation, we chose a complementary approach to investigate the impact of autoimmunity on immune signatures. Because the evolution of autoimmune diseases such as MS is a gradual process, we hypothesized that an analysis of MS-related effects on immune traits would be most promising at the earliest stages of the disease as this avoids any confounding effects related to disease stage and treatment. This concept of a gradual evolution has gained much interest in recent years $(32,33)$. In our cohort, the clinically healthy cotwins have a maximum familial risk for developing MS (34). Indeed, we were able to identify a subcohort of clinically healthy cotwins who showed signs of subclinical neuroinflammation (8). This offered us the unique possibility to study potential MS-related effects on each immune trait by comparing the degree of correlation in twin pairs who were clinically and subclinically discordant, to those where the healthy twin exhibited subclinical signs of prodromal MS. By this approach, we were indeed able to identify a pattern of potential MS-associated adaptive immune traits, especially within CD $4^{+}$ effector T cell subsets. This finding supports the concept that MS is initiated and orchestrated by antigen-driven T cells $(6,7)$.

Notably, we had the opportunity to analyze flow cytometry data from a second independent cohort of untreated patients with either clinically isolated syndrome or early RRMS to evaluate whether the immune traits identified in our prodromal MS approach might be differentially expressed in early untreated MS patients. Indeed, several of our identified immune traits were found to be differentially expressed, in particular within the Th1 and Th17 effector populations. Despite using distinct approaches to study MS-related changes of immune signatures in incipient disease in both cohorts, our analysis revealed that very early disease stages were particularly associated with changes in peripheral immune signatures.

Interestingly, some of these immune traits identified in our systems approach had already been implicated in MS pathophysiology, either based on preclinical findings from animal models of MS or differential expression patterns using focused hypothesisdriven approaches in smaller cohorts of MS patients (35). For example, Th17 cells and a specific subset, termed Th17.1 cells, have both been shown to be highly encephalitogenic in EAE models and found to be increased in the peripheral blood of early MS patients (36-38). However, in contrast to these previous approaches, which investigated single individual candidates in a hypothesis-driven fashion, we now employed an unbiased approach that allowed us to identify MS-associated signatures in distinct immune cell populations of our twin cohort. Our findings are in line with a recent study employing a combination of single-cell mass cytometry (CyTOF) with machine-learning algorithms to identify a T-helper cell signature in MS patients (39).

It is interesting to note that our approach did not reveal a significant impact of MS on B cell traits. This finding seemingly contradicts the increasingly acknowledged role of B cells in MS highlighted by the success of B cell-depleting treatment strategies both in RRMS and primary progressive MS (40, 41). However, we acknowledge that our flow cytometry panels captured a higher number of $\mathrm{T}$ cell as compared to $\mathrm{B}$ cell traits, which in contrast to B cell traits include functionality assessments, resulting in a higher power for detection of alterations in the $\mathrm{T}$ cell compartment. Furthermore, our analysis in Fig. $2 B$ revealed that $\mathrm{B}$ cell traits might be particularly susceptible to immune treatment effects. It should be pointed out that our regression analysis also suggested more pronounced MS effects on a few selected B cell traits, for example regulatory B cells and memory B cells. In addition, for selected antigen-presenting monocyte and DC populations, an individually higher MS influence could be noted, which is in line with other publications highlighting the potential role of specific myeloid cell subsets in CNS autoimmunity $(42,43)$.

The observed predominance of MS-associated adaptive immune traits in our correlation approach might at least partly be related to the fact that our phenotyping strategy overall covered more adaptive (102 traits) than innate (39 traits) immune traits. We therefore cannot rule out that a more detailed investigation of innate immune traits might reveal additional MS-associated features in these populations, since innate populations are increasingly acknowledged as key players in the pathogenesis of MS (44-46).

From a more general perspective, our approach illustrates that a stringent control for interindividual heterogeneity by use of a twin design combined with a focus on very early disease stages, thus minimizing disease-related heterogeneity, represents a powerful strategy to elucidate changes of the peripheral immunome in human autoimmunity in a comprehensive fashion.

By analyzing subjects with subclinical (prodromal) signs of neuroinflammation we were able to uncover MS-associated immune signatures that would otherwise have escaped detection. We are aware of the relatively small sample size, but this is outbalanced by the distinct advantages of our twin approach. It should be mentioned that, albeit our deep-immune phenotyping panel encompasses a large number of immune traits, we cannot rule out that it does not fully capture more sophisticated functional immune 
features, such as antigen recognition, functional exhaustion, active suppression, or other tolerance mechanisms.

In particular, in vitro stimulation of $\mathrm{T}$ cells with relevant target autoantigens and ex vivo staining with antigenic-peptide-MHC multimers might have revealed stronger MS-associated "signatures." However, apart from technical hurdles, validated target antigens are lacking in MS $(6,7)$. This contrasts with other autoimmune diseases of the CNS where the target antigens are well established, for example, neuromyelitis optica spectrum disease (characterized by antiaquaporin-4-antibodies) and myelinoligodendrocyte glycoprotein (MOG)-antibody associated autoimmune disorder (characterized by anti MOG-antibodies) (47).

Another limitation is that with this approach we analyzed only blood and not CSF. While CSF analysis likely could provide important additional information, it poses practical and ethical obstacles because it would imply systematic invasive CSF sampling from healthy individuals. From a scientific point of view, however, analysis of immune signatures in secondary lymphatic structures as well as in CSF from our twin pairs would be highly interesting as these might more closely reflect target-organ and autoimmunity-associated changes of immune cell traits. Indeed, in a small cohort of monozygotic twins with prodromal MS, single-cell RNA sequencing of CSF cells revealed alterations in the adaptive immune compartment, such as clonal expansion of tissue-resident memory $\mathrm{CD}^{+} \mathrm{T}$ cells, plasma blasts, as well as $\mathrm{CD}^{+}{ }^{+} \mathrm{T}$ cells, suggesting an involvement of all major components of the adaptive immune system (8).

Taking these data together, by controlling for the dominant influence of genetic and local environmental factors, and by focusing on incipient MS, we were able to reveal peripheral MSrelated immune signatures pointing to a pivotal role of effector $\mathrm{CD} 4^{+} \mathrm{T}$ cells during disease initiation. Our study underscores the crucial importance of genetic and environmental heterogeneity as an obstacle in the search for disease-related immune signatures. Our in-depth analysis of healthy as well as cotwins with prodromal MS helped us to identify patterns of MSassociated immune alterations, some of which could be corroborated in an independent cohort of early, untreated RRMS patients. In future studies it will be of great interest to correlate such MS-linked variations of immune phenotypes with the emerging genetic and epigenetic risk profile of MS.

\section{Materials and Methods}

Study Cohorts. Twins were recruited between May 2012 and February 2016 in Germany. Inclusion criteria for study participation were met if only one cotwin of monozygotic twins had an MS diagnosis according to the revised McDonald criteria $(10,21)$. Exclusion criteria were infection, as well as treatment with antibiotics or high dose intravenous glucocorticosteroids within 3 mo prior to sampling. Monozygotic twin pairs clinically discordant for MS visited the outpatient department at the Institute of Clinical Neuroimmunology in Munich for a detailed interview, neurological examination, MRI investigations, blood sampling, and optional CSF sampling. To confirm MS diagnosis, medical records including MRI scans were obtained and reviewed. Exclusion criteria for the present analysis were a current disease modifying treatment (DMT) with fingolimod, alemtuzumab, dimethylfumarate, or teriflunomide, resulting in 43 of 53 monozygotic twin pairs eligible for analysis (see Table 1 for basic clinical characteristics). Based on the analysis in a healthy twin cohort by Brodin et al. (25), showing a profound influence of CMV serostatus on immune signatures, we determined CMV serostatus ( $n=42$ pairs) in our cohort and it was included in our modeling. Notably, Epstein-Barr virus (EBV) serostatus was determined ( $n=36$ pairs); however, we identified only one single HD twin being EBV seronegative.

At the time of blood collection, 22 MS-affected cotwins did not receive any DMT, 21 MS-affected cotwins were treated with different DMT, including IFN- $\beta(n=14)$, natalizumab $(n=4)$, or glatiramer acetate $(n=3)$. MRI and CSF sampling detected subtle signs of inflammation in 10 of 43 clinically healthy cotwins, which was classified as SCNI potentially reflecting prodromal MS in this high-risk cohort (see details in Dataset S1) $(8,20,48-53)$. In order to provide a clear-cut dichotomization of both ends of the spectrum (i.e., healthy vs. SCNI cotwins), healthy cotwins in which MRI-data were missing or inconclusive, and CSF data to either confirm or rule out SCNI was lacking were excluded from this subgroup analysis $(n=19)$. Monozygosity was confirmed by genotyping 17 highly polymorphic microsatellite markers and by next-generation sequencing of 33 SNPs.

The Muenster early MS cohort consists of 115 early treatment-naïve MS patients diagnosed according to the revised McDonald criteria with a disease onset $<36$ mo. In parallel, 71 age- and sex-matched healthy controls were assessed (Table 1).

The study was approved by the local ethics committees of the LudwigMaximilians University of Munich (ethics approval project number 267-13) and of the University of Muenster (ethics approval project number 2010262-f-S). All participants gave written informed consent, according to the principles of the Declaration of Helsinki.

Blood Sampling and PBMC Preparation. Blood samples of study participants were collected in EDTA-containing tubes. To exclude sample collection bias, blood samples were drawn from each twin pair before meals and at the same time on the same day. PBMCs were isolated as described before by density gradient centrifugation with Lymphoprep (Stemcell Technologies) and cryopreserved in liquid nitrogen using serum-free cryopreservation medium (CTL-Cryo ABC Media Kit, Immunospot) in concentrations of $1 \times 10^{7}$ cells/mL (44).

Immune Phenotyping by Flow Cytometry. For flow cytometry analysis 13 panels consisting of 9 to 10 fluorescence markers were measured (cellular functional immune phenotyping matrix). Gating strategies and identification of populations are depicted in Dataset S2. All samples were stained and measured over a short period of $4 \mathrm{wk}$ by two experienced technicians on the same flow cytometer. Cotwins were stained and measured on the same day to minimize acquisition variability. To label cell surface molecules, cryoconserved PBMCs were reconstituted in PBS/0.5\% BSA 2 mM EDTA containing fluorochrome-conjugated antibodies and incubated for $30 \mathrm{~min}$ at $4{ }^{\circ} \mathrm{C}$ or $37^{\circ} \mathrm{C}$. For intracellular staining, cells were fixed and permeabilized for $20 \mathrm{~min}$ at $4^{\circ} \mathrm{C}$ with Cytofix/CytopermTM working solution (eBioscience) according to the manufacturer's instructions, and subsequently stained with intracellular fluorochrome-conjugated antibodies for $30 \mathrm{~min}$ at $4{ }^{\circ} \mathrm{C}$. To determine intracellular cytokine secretion, PBMCs were restimulated in X-vivo (Lonza) $\pm 10 \mu \mathrm{L} / \mathrm{mL}$ LAC (Leukocyte Activation Mixture; PMA, Ionomycin, Brefeldin A; BD Pharmingen) for $4 \mathrm{~h}$ at $37{ }^{\circ} \mathrm{C} / 5 \% \mathrm{CO}_{2}$. Working antibody concentrations are depicted in Dataset S3. All flow cytometry data were acquired by Navios (twin cohort) and Cytoflex (Beckman Coulter, validation cohort) flow cytometers and analyzed by Kaluza analysis software 1.5 (Beckman Coulter) and GraphPad Prism 6. Data analysis was performed by two independent scientists (four-eyes principle).

Single-Cell Multiparameter Visualization. We used an unbiased approach to visualize flow cytometry data on a multiparameter single-cell basis. We analyzed 13 flow cytometry panels consisting of 9 to 10 fluorescence and 2 scatter parameters using viSNE, a Matlab-based unbiased nonlinear dimension reduction visualization tool. The bh-SNE algorithm maps single-cell data points from high-dimensional to $2 \mathrm{D}$ space, emphasizing local rather than global similarities by minimizing the difference in pairwise similarities between points $(54,55)$. The generated List Mode Data (LMD) files were converted into Flow Cytometry Standard (FCS) files using an R studio-based script provided by Beckman Coulter. Prior to clustering, we applied a linear nor malization algorithm to reduce sample-to-sample acquisition variability (56 57). Data points of twin FCS files were randomly subsampled, limiting the data point size to 1,000 to 2,000 points per individual. To promote divergence and avoid "crowding" of clusters, data point size was limited to 100,000 points in total. Samples were merged and subsequently mapped into a 2D bh-SNE plot We applied the PhenoGraph algorithm to identify local, single-cell-based similarities (cluster) (15). PhenoGraph was run using a setting of $k$-nearest neighbors between 300 and 500 based on investigator expertise and a Euclidean distance metric to yield sample-level subpopulation clusters ranging from 1 to $10 \%$ per panel. This setting generated 19 to 34 distinct immune cell clusters per panel. This adds up to an overall number of 352 cluster (355 clusters in the treatment naïve analysis in SI Appendix, Fig. S1C), which determined the unique immune signature of the individual twins allowing for indepth comparison of immune status in the twin pairs. A heatmap illustrates twofold change of reduced (green) and increased (red) percental differences of clusters between the healthy and MS conditions (e.g., Fig. 1A).

Determination of Biological Distance. Conventionally gated populations of the sub collectives $\mathrm{CD} 4^{+}$were divided into activated, effector, and other subsets (depicted in Dataset S4) according to their immunological phenotype (total 
number of investigated populations: $\mathrm{CD}^{+}$activated subset, $n=6 ; \mathrm{CD}^{+}$ other subset, $n=7$; $C D 4^{+}$effector subset, $n=13$ ). Frequency values per population were normalized and clustered by the t-SNE algorithm using the Rtsne package in R. Biological distance was determined based on the Euclidian distance of t-SNE coordinates $(16,18,58)$. To account for clustering variability and assess analysis robustness, the analysis was performed three times (without a seed) and the mean was used to determine the biological distance. Only twin pairings with complete datasets per parameter were included into this analysis. Statistical significance was determined with GraphPad Prism 6 by the Mann-Whitney $U$ test. ${ }^{*} P \leq 0.05 ;{ }^{*} P \leq 0.01$.

Regression Analysis. Regression analyses were performed using SAS v9.4 (SAS Institute) and R v3.6.1 (59). For each immune parameter, a linear mixed model was fitted including age, gender, CMV, and disease status (MS yes/no) as fixed effects as well as a random intercept in order to account for the correlation within twin pairs (SAS, proc mixed). Based on the mixed models, marginal and conditional $R^{2} s$ were computed according to Nakagawa and Schlielzeth (60). The authors indicate the proportion of variance explained based on the fixed effects (marginal $R^{2}$ ) and the fixed plus the random effects (conditional $R^{2}$ ) (i.e., also accounting for the correlation within pairs of twins). ICCs were calculated by dividing the variance estimate of the random effect by the sum of this variance and the residual variance. In addition, correlation coefficients (Pearson/Spearman) were calculated to assess the correlation within twin pairs for each parameter. Additionally, the proportion of variance explained by the different fixed effects was estimated using the relaimpo package (Img metric) (61). In order to account for correlation within twin pairs, a workaround for clustered data by specifying a survey design was used as presented in the package manual.

Mixed-model analyses were repeated for the two separate twin cohorts with healthy cotwins and SCNI cotwins. The ICCs from these models (adjusted

1. B. Engelhardt, P. Vajkoczy, R. O. Weller, The movers and shapers in immune privilege of the CNS. Nat. Immunol. 18, 123-131 (2017).

2. M. Sospedra, R. Martin, Immunology of multiple sclerosis. Semin. Neurol. 36, 115-127 (2016).

3. A. J. Thompson, S. E. Baranzini, J. Geurts, B. Hemmer, O. Ciccarelli, Multiple sclerosis. Lancet 391, 1622-1636 (2018).

4. A. Ben-Nun, H. Wekerle, I. R. Cohen, Pillars article: The rapid isolation of clonable antigen-specific $\mathrm{T}$ lymphocyte lines capable of mediating autoimmune encephalomyelitis. Eur J Immunol. 1981.11: 195-199. J. Immunol. 198, 3384-3388 (2017).

5. S. S. Zamvil, L. Steinman, The T lymphocyte in experimental allergic encephalomyelitis. Annu. Rev. Immunol. 8, 579-621 (1990).

6. R. Hohlfeld, K. Dornmair, E. Meinl, H. Wekerle, The search for the target antigens of multiple sclerosis, part 1: Autoreactive CD4+ T lymphocytes as pathogenic effectors and therapeutic targets. Lancet Neurol. 15, 198-209 (2016).

7. R. Hohlfeld, K. Dornmair, E. Meinl, H. Wekerle, The search for the target antigens of multiple sclerosis, part 2: CD8+ T cells, B cells, and antibodies in the focus of reversetranslational research. Lancet Neurol. 15, 317-331 (2016).

8. E. Beltrán et al., Early adaptive immune activation detected in monozygotic twins with prodromal multiple sclerosis. J. Clin. Invest. 129, 4758-4768 (2019).

9. L. Steinman, No quiet surrender: Molecular guardians in multiple sclerosis brain J. Clin. Invest. 125, 1371-1378 (2015)

10. A. J. Thompson et al., Diagnosis of multiple sclerosis: 2017 revisions of the McDonald criteria. Lancet Neurol. 17, 162-173 (2018).

11. C. Lebrun, The radiologically isolated syndrome. Rev. Neurol. 171, 698-706 (2015).

12. S. E. Baranzini, J. R. Oksenberg, The genetics of multiple sclerosis: From 0 to 200 in 50 years. Trends Genet. 33, 960-970 (2017).

13. L. Lohmann et al., Immune cell profiling during switching from natalizumab to fingolimod reveals differential effects on systemic immune-regulatory networks and on trafficking of non-T cell populations into the cerebrospinal fluid-results from the ToFingo Successor Study. Front. Immunol. 9, 1560 (2018).

14. L. Klotz et al., Teriflunomide treatment for multiple sclerosis modulates T cell mitochondrial respiration with affinity-dependent effects. Sci. Transl. Med. 11, eaao5563 (2019).

15. J. H. Levine et al., Data-driven phenotypic dissection of AML reveals progenitor-like cells that correlate with prognosis. Cell 162, 184-197 (2015).

16. L. J. P. van der Maaten, Accelerating t-SNE using tree-based algortihms. J. Mach Learn. Res. 15, 3221-3245 (2014).

17. M. Roederer et al., The genetic architecture of the human immune system: A bioresource for autoimmunity and disease pathogenesis. Cell 161, 387-403 (2015).

18. E. J. Carr et al., The cellular composition of the human immune system is shaped by age and cohabitation. Nat. Immunol. 17, 461-468 (2016)

19. E. Patin et al.; Milieu Intérieur Consortium, Natural variation in the parameters of innate immune cells is preferentially driven by genetic factors. Nat. Immunol. 19, 302-314 (2018).

20. Z. Xia et al., Assessment of early evidence of multiple sclerosis in a prospective study of asymptomatic high-risk family members. JAMA Neurol. 74, 293-300 (2017).

21. C. H. Polman et al., Diagnostic criteria for multiple sclerosis: 2010 revisions to the McDonald criteria. Ann. Neurol. 69, 292-302 (2011). for age, gender, and (MV) were compared using a mixed model accounting for the dependence of immune parameters. Therefore, the correlation of immune parameters was calculated based on the whole cohort using the marginal residuals from linear mixed models (i.e., accounting for age, gender, and (MV). Because of the correlation of residuals within twin pairs, the residuals were averaged for each pair of twins (62). The correlation between immune parameters was then estimated using Spearman's rank correlation coefficient. The sample correlation matrix was implemented in the mixed model by applying the Ime4qtl package in order to specify the covariance matrix for the random effects (63). No adjustment for multiple testing was applied (i.e., all significance levels are to be understood as local significance levels). Therefore, inferential statistics are intended to be exploratory (hypotheses generating), not confirmatory.

Data Availability. All study data are included in the article, the SI Appendix, and Datasets S1-S8. Full protected access of flow cytometry data will be granted to collaborating scientists who are willing to complete an "MS-TWIN Data Access Agreement" and an "MS-TWIN Data Access Application form."

ACKNOWLEDGMENTS. We thank all subjects who participated in this study; and Katja Anslinger, Department of Forensic Medicine, Ludwig-Maximilians University Munich, for monozygosity determination. The study was supported by the German Research Council through CRC128 A05, CRC128 A08, CRC128 Z02; the Munich Cluster for Systems Neurology (SyNergy EXC 2145, project 390857198); the Gemeinnützige Hertie Stiftung; Bavarian Association and National Association of the German MS society; Dr. Leopold and Carmen Ellinger Foundation; and the Association "Verein zur Therapieforschung für MS Kranke e.V." F.B. is supported by the National Institute for Health Research Biomedical Research Centre at University College London Hospitals.

22. J. S. Hale, T. E. Boursalian, G. L. Turk, P. J. Fink, Thymic output in aged mice. Proc. Natl. Acad. Sci. U.S.A. 103, 8447-8452 (2006).

23. M. Mangino, M. Roederer, M. H. Beddall, F. O. Nestle, T. D. Spector, Innate and adaptive immune traits are differentially affected by genetic and environmental factors. Nat. Commun. 8, 13850 (2017).

24. R. Aguirre-Gamboa et al., Differential effects of environmental and genetic factors on T and B cell immune traits. Cell Rep. 17, 2474-2487 (2016).

25. P. Brodin et al., Variation in the human immune system is largely driven by nonheritable influences. Cell 160, 37-47 (2015).

26. V. Lagou et al., Genetic architecture of adaptive immune system identifies key immune regulators. Cell Rep. 25, 798-810.e6 (2018).

27. V. Orrù et al., Genetic variants regulating immune cell levels in health and disease. Cell 155, 242-256 (2013).

28. E. Sharon et al., Genetic variation in MHC proteins is associated with T cell receptor expression biases. Nat. Genet. 48, 995-1002 (2016).

29. N. Y. Souren et al., DNA methylation signatures of monozygotic twins clinically discordant for multiple sclerosis. Nat. Commun. 10, 2094 (2019).

30. L. Belbasis, V. Bellou, E. Evangelou, J. P. Ioannidis, I. Tzoulaki, Environmental risk factors and multiple sclerosis: An umbrella review of systematic reviews and metaanalyses. Lancet Neurol. 14, 263-273 (2015).

31. T. Olsson, L. F. Barcellos, L. Alfredsson, Interactions between genetic, lifestyle and environmental risk factors for multiple sclerosis. Nat. Rev. Neurol. 13, 25-36 (2017).

32. J. M. Wijnands et al., Five years before multiple sclerosis onset: Phenotyping the prodrome. Mult. Scler. 25, 1092-1101 (2019).

33. G. Giovannoni, How long is the presymptomatic phase of multiple sclerosis? Mult. Scler. Relat. Disord. 7, 12-13 (2016).

34. S. E. Baranzini et al., Genome, epigenome and RNA sequences of monozygotic twins discordant for multiple sclerosis. Nature 464, 1351-1356 (2010)

35. C. A. Dendrou, L. Fugger, M. A. Friese, Immunopathology of multiple sclerosis. Nat. Rev. Immunol. 15, 545-558 (2015).

36. Y. Cao et al., Functional inflammatory profiles distinguish myelin-reactive T cells from patients with multiple sclerosis. Sci. Transl. Med. 7, 287 ra74 (2015).

37. J. van Langelaar et al., T helper 17.1 cells associate with multiple sclerosis disease activity: Perspectives for early intervention. Brain 141, 1334-1349 (2018)

38. V. Brucklacher-Waldert, K. Stuerner, M. Kolster, J. Wolthausen, E. Tolosa, Phenotypical and functional characterization of T helper 17 cells in multiple sclerosis. Brain 132, 3329-3341 (2009).

39. E. Galli et al., GM-CSF and CXCR4 define a T helper cell signature in multiple sclerosis. Nat. Med. 25, 1290-1300 (2019).

40. S. L. Hauser et al.; OPERA I and OPERA II Clinical Investigators, Ocrelizumab versus interferon beta-1a in relapsing multiple sclerosis. N. Engl. J. Med. 376, 221-234 (2017).

41. X. Montalban et al:; ORATORIO Clinical Investigators, Ocrelizumab versus placebo in primary progressive multiple sclerosis. N. Engl. J. Med. 376, 209-220 (2017).

42. A. Waschbisch et al., Pivotal role for CD16+ monocytes in immune surveillance of the central nervous system. J. Immunol. 196, 1558-1567 (2016).

43. S. Mundt et al., Conventional DCs sample and present myelin antigens in the healthy CNS and allow parenchymal T cell entry to initiate neuroinflammation. Sci. Immunol. 4, eaau8380 (2019). 
44. C. C. Gross et al., Impaired NK-mediated regulation of T-cell activity in multiple sclerosis is reconstituted by IL-2 receptor modulation. Proc. Natl. Acad. Sci. U.S.A. 113, E2973-E2982 (2016).

45. L. Van Kaer, J. L. Postoak, C. Wang, G. Yang, L. Wu, Innate, innate-like and adaptive lymphocytes in the pathogenesis of MS and EAE. Cell. Mol. Immunol. 16, 531-539 (2019).

46. I. Tahrali et al., Dysfunction of $\mathrm{CD}^{-} \mathrm{CD} 16^{+} \mathrm{CD} 56^{\text {dim }}$ and $\mathrm{CD}^{-} \mathrm{CD} 16^{-} \mathrm{CD} 56^{\text {bright }} \mathrm{NK}$ cell subsets in RR-MS patients. Clin. Immunol. 193, 88-97 (2018).

47. S. Mader, T. Kümpfel, E. Meinl, Novel insights into pathophysiology and therapeutic possibilities reveal further differences between AQP4-IgG- and MOG-IgG-associated diseases. Curr. Opin. Neurol. 33, 362-371 (2020).

48. N. Makhani et al.; Observatoire Francophone de la Sclérose en Plaques (OFSEP), Société Francophone de la Sclérose en Plaques (SFSEP), the Radiologically Isolated Syndrome Consortium (RISC) and the Pediatric Radiologically Isolated Syndrome Consortium (PARIS), Oligoclonal bands increase the specificity of MRI criteria to predict multiple sclerosis in children with radiologically isolated syndrome. Mult. Scler. J. Exp. Transl. Clin. 5, 2055217319836664 (2019).

49. T. Hansen et al., Concordance for multiple sclerosis in Danish twins: An update of a nationwide study. Mult. Scler. 11, 504-510 (2005).

50. T. Hansen et al., Risk for multiple sclerosis in dizygotic and monozygotic twins. Mult. Scler. 11, 500-503 (2005).

51. J. W. Thorpe et al., British Isles survey of multiple sclerosis in twins: MRI. J. Neurol. Neurosurg. Psychiatry 57, 491-496 (1994).

52. N. De Stefano et al., Imaging brain damage in first-degree relatives of sporadic and familial multiple sclerosis. Ann. Neurol. 59, 634-639 (2006).
53. T. Gabelic et al., Prevalence of radiologically isolated syndrome and white matter signal abnormalities in healthy relatives of patients with multiple sclerosis. AJNR Am. J. Neuroradiol. 35, 106-112 (2014).

54. L. Sgier, R. Freimann, A. Zupanic, A. Kroll, Flow cytometry combined with viSNE for the analysis of microbial biofilms and detection of microplastics. Nat. Commun. 7 , 11587 (2016).

55. A. D. Amir et al., viSNE enables visualization of high dimensional single-cell data and reveals phenotypic heterogeneity of leukemia. Nat. Biotechnol. 31, 545-552 (2013).

56. F. J. Hartmann et al., High-dimensional single-cell analysis reveals the immune signature of narcolepsy. J. Exp. Med. 213, 2621-2633 (2016).

57. S. M. Gibbons, C. Duvallet, E. J. Alm, Correcting for batch effects in case-control microbiome studies. PLoS Comput. Biol. 14, e1006102 (2018).

58. J. H. Krijthe, Rtsne: T-Distributed Stochastic Neighbor Embedding using a Barnes-Hut Implementation, R package version 0.15 (2015). https://github.com/jkrijthe/Rtsne. Accessed 7 October 2019.

59. R Core Team, R: A Language and Environment for Statistical Computing (R Foundation for Statistical Computing, Vienna, Austria, 2018).

60. H. S. Nakagawa, H. Schielzeth, A general and simple method for obtaining $R^{2}$ from generalized linear mixed-effects models. Methods Ecol. Evol. 4, 133-142 (2013).

61. U. Grömping, Relative importance for linear regression in R: The package relaimpo. J. Stat. Softw., 10.18637/jss.v017.i01 (2006).

62. J. M. Bland, D. G. Altman, Calculating correlation coefficients with repeated observations: Part 2-Correlation between subjects. BMJ 310, 633 (1995).

63. A. Ziyatdinov et al., Ime4qtl: Linear mixed models with flexible covariance structure for genetic studies of related individuals. BMC Bioinf. 19, 68 (2018) 